\title{
LONG TIME EXISTENCE OF HYPERBOLIC RICCI-BOURGUIGNON FLOW ON RIEMANNIAN SURFACES
}

\author{
Mehrad Mohammadi ${ }^{1}$ And Shahroud Azami $^{2}$ \\ ${ }^{1}$ Department of pure Mathematics, Faculty of Science, Imam Khomeini \\ International University, Qazvin, Iran, mehrad76@gmail.com \\ ${ }^{2}$ Department of pure Mathematics, Faculty of Science, Imam Khomeini \\ International University, Qazvin, Iran, azami@sci.ikiu.ac.ir
}

\begin{abstract}
We consider the hyperbolic Ricci-Bourguignon flow(HRBF) equation on Riemannian surfaces and we find a sufficient and necessary condition to this flow has global classical solution. Also, we show that the scalar curvature of the solution metric $g_{i j}$ convergence to the flat curvature.
\end{abstract}

Key words and Phrases: Hyperbolic geometric flow; quasilinear hyperbolic equation; strict hyperbolicity

\section{INTRODUCTION}

Let $(M, g)$ be an n-dimensional complete Riemannian manifold with Riemannian metric $g_{i j}$. The general variation equation

$$
\frac{\partial^{2} g_{i j}}{\partial t^{2}}+2 R_{i j}+\mathcal{F}\left(g, \frac{\partial g}{\partial t}\right)=0
$$

was introduced by Kong and Liu ([4]) and called the generalized hyperbolic geometric flow (denoted by HGF). Here $\mathcal{F}$ are some smooth functions of the Riemannian metric and its first derivative with respect to $t$, and we consider $R_{i j}$ as the components of Ricci curvature tensor. Liu and Zhang in ([8]) have shown that the hyperbolic geometric flow (HGF) has global classical solution on Riemannian surfaces. In this paper, we would like to prove that the global solution of hyperbolic Ricci-Bourguignon flow (HRBF) exists on Riemannian surfaces.

The present work investigates the variation of a Riemannian metric $g_{i j}$ on a Riemannian surface $M$ by its Ricci curvature tensor $R_{i j}$ and scalar curvature $R$ under

2020 Mathematics Subject Classification: 58J45, 58J47

Received: 25-06-2019, accepted: 10-05-2020. 
the following equation

$$
\frac{\partial^{2} g_{i j}}{\partial t^{2}}=-2 R_{i j}+2 \rho R g_{i j}
$$

where $\rho$ is a real constant. When $\rho=0$, this equation is hyperbolic geometric flow and the global existence and blowup phenomenon of smooth solutions to this flow on Riemannian surface have been investigated in [8]. The Ricci-Bourguignon flow is $\frac{\partial g_{i j}}{\partial t}=-2 R_{i j}+2 \rho R g_{i j}$ and the short time existence and uniqueness for solution to the Ricci-Bourguignon flow on $[0, T)$ were showed by Catino et al ([1]) for $\rho<\frac{1}{2(n-1)}$.

This study regards the initial metric as follows

$$
d s^{2}=u_{0}(x)\left(d x^{2}+d y^{2}\right) \quad \text { at } t=0
$$

on a surface of topological type $\mathbb{R}^{2}$, where $u_{0}(x)$ is a function from $C^{2}$ class with bounded $C^{2}$ norm and the following inequality is hold

$$
0<k \leq u_{0}(x) \leq m<\infty
$$

where $k$ and $m$ are positive constants.

Since all the information about curvature is contained in the scalar curvature function $R$, we can simplify the HRBF equation on this surface. According to our notation, $R=2 K$, where $K$ denotes Gauss curvature and also the Ricci curvature is given by $R_{i j}=\frac{1}{2} R g_{i j}$, so the (HRBF) equation simplifies to

$$
\frac{\partial^{2} g_{i j}}{\partial t^{2}}=-R(1-2 \rho) g_{i j}
$$

At least locally the metric for a surface can be written as $g_{i j}=u(t, x, y) \delta_{i j}$, where $u(t, x, y)>0$, and $\delta_{i j}$ is Kronecker's symbol. Hence, we have

$$
R=-\frac{\Delta \ln u}{u}
$$

as a result, the aforementioned equation (5) reduces to $u_{t t}-(1-2 \rho) \Delta \ln u=0$.

The initial data $u_{0}(x)$ depends only on $x$ and not $y$; thus, we can consider the Cauchy problem as below

$$
\left\{\begin{array}{l}
u_{t t}-(1-2 \rho)(\ln u)_{x x}=0 \\
u=u_{0}(x), \quad \text { at } t=0 \\
u_{t}=u_{1}(x), \quad \text { at } t=0
\end{array}\right.
$$

where $u_{1}(x) \in C^{1}$ with bounded $C^{1}$ norm. By using the transformation

$$
\phi=\ln u,
$$

Kong and Liu in ([5]) proved a theorem as follows

Theorem 1.1. Suppose that $u_{1}(x) \geq\left|u_{0}^{\prime}(x)\right| / \sqrt{u_{0}(x)}$ for all $x \in \mathbb{R}$. Then, the Cauchy problem (7) admits a unique global solution for all $t \in \mathbb{R}$.

Moreover, if $u_{1}(x) \equiv u_{0}^{\prime}(x) / \sqrt{u_{0}(x)}$, and there exists a point $x_{0} \in \mathbb{R}$ such 
that $u_{0}^{\prime}\left(x_{0}\right)<0$, then the Cauchy problem (7) admits a unique classical solution only in $[0, T) \times \mathbb{R}$, where

$$
T=-\frac{2}{i n f_{x}\left\{u_{0}^{\prime}(x) u_{0}^{-3 / 2}(x)\right\}} .
$$

The following theorem will proven without using (6) in our investigate.

Theorem 1.2. Let

$$
u_{1}(x)+\frac{u_{0}^{\prime}(x)}{\sqrt{u_{0}(x)}} \geq 0 \quad \text { at all } x \in \mathbb{R},
$$

and

$$
u_{1}(x)-\frac{u_{0}^{\prime}(x)}{\sqrt{u_{0}(x)}} \geq 0 \quad \text { at all } x \in \mathbb{R} .
$$

Hence, the Cauchy problem (7) has a unique global solution for all $t \in \mathbb{R}$.

Theorem 1.3. If a point $x_{0} \in \mathbb{R}$ exists, which satisfy

$$
u_{1}\left(x_{0}\right)+\frac{u_{0}^{\prime}\left(x_{0}\right)}{\sqrt{u_{0}\left(x_{0}\right)}}<0
$$

or there exists a point $x_{0} \in \mathbb{R}$, such that

$$
u_{1}\left(x_{0}\right)-\frac{u_{0}^{\prime}\left(x_{0}\right)}{\sqrt{u_{0}\left(x_{0}\right)}}<0
$$

thus, the Cauchy problem (7) has a unique classical solution only in $[0, T) \times \mathbb{R}$.

Note. Based on Theorem 1.2, we can conclude the Cauchy problem

$$
\begin{cases}\frac{\partial^{2} g_{i j}}{\partial t^{2}}=-2 R_{i j}+2 \rho R g_{i j}, & \text { for } i, j=1,2 \\ g_{i j}=u_{0}(x) \delta_{i j}, & \text { for } t=0 \\ \frac{\partial g_{i j}}{\partial t}=u_{1}(x) \delta_{i j}, & \text { for } t=0 \text { and } i, j=1,2 .\end{cases}
$$

has a unique smooth solution for all $t \in \mathbb{R}$. Besides we can consider the solution metric $g_{i j}$ as below

$$
g_{i j}=u(x, t) \delta_{i j} \quad \text { for } i, j=1,2 .
$$

We will prove the above mentioned Theorems 1.2 and 1.3 , in the subsequent sections (3 and 4, respectively). Moreover, using Theorem 1.2, the following theorem will be proven in Section 5 .

Theorem 1.4. Let be

$$
\text { inf } f_{x}\left\{u_{1}(x)+\frac{u_{0}^{\prime}(x)}{\sqrt{u_{0}(x)}}\right\}>0 \quad \text { and } \quad \text { inf } f_{x}\left\{u_{1}(x)-\frac{u_{0}^{\prime}(x)}{\sqrt{u_{0}(x)}}\right\}>0 .
$$

Hence, a unique classical solution of (1) is exist as the form (14) for all time. Furthermore, the scalar curvature $R(x, t)$ relates to the solution metric $g_{i j}$ admits

$$
R(x, t) \rightarrow 0 \quad \text { as } t \rightarrow+\infty,
$$

and $R(x, t) \leq k_{1}$ for all $(t, x) \in \mathbb{R}^{+} \times \mathbb{R}$, where $k_{1}$ is a positive constant and independent of $t$ and $x$. 


\section{Preliminaries}

In this section we require only to discuss the classical solution on $t \geq 0$. The result for $t \leq 0$ can be easily obtained.

Suppose that

$$
u_{t}=v \quad \text { and } \quad u_{x}=w .
$$

Thus, from the above equations and Cauchy problem (7) we have

$$
u_{t}=v, \quad w_{t}-v_{x}=0, \quad \text { and } \quad v_{t}-\left(\frac{1-2 \rho}{u}\right) w_{x}=(2 \rho-1) \frac{w^{2}}{u^{2}} .
$$

Eigenvalues of equations (17) can be easily calculated as follows

$$
\lambda_{1}=-\lambda, \quad \lambda_{2}=0, \quad \lambda_{3}=\lambda, \quad \lambda=\sqrt{\frac{1-2 \rho}{u}}
$$

and we have the matrices $L(U)$ and $R(U)$ (where $U=(u, w, v)$ ) of left and right eigenvectors, respectively as below

$$
\begin{aligned}
& L(U)=\left(\begin{array}{l}
l_{1}(U) \\
l_{2}(U) \\
l_{3}(U)
\end{array}\right)=\left(\begin{array}{ccc}
0 & \lambda & 1 \\
1 & 0 & 0 \\
0 & -\lambda & 1
\end{array}\right), \\
& R(U)=\left(r_{1}(u), r_{2}(u), r_{3}(u)\right)=\left(\begin{array}{ccc}
0 & 1 & 0 \\
\lambda & 0 & -\lambda \\
1 & 0 & 1
\end{array}\right) .
\end{aligned}
$$

Equation system (17) is a linear degenerate strict hyperbolic system because of $\nabla \lambda_{i}(U) r_{i}(U) \equiv 0$ for $i=1,2,3$.

Define $p$ and $q$ as follows

$$
p=v+\lambda w \quad \text { and } \quad q=v-\lambda w .
$$

Lemma 2.1. $p$ and $q$ satisfy the following equations:

$$
\begin{gathered}
p_{t}-\lambda p_{x}=\frac{1}{4(1-2 \rho)} \lambda^{2}(q-p) p, \\
u_{t}=\frac{1}{2}(p+q), \\
q_{t}+\lambda q_{x}=\frac{1}{4(1-2 \rho)} \lambda^{2}(p-q) q .
\end{gathered}
$$

Proof. By differentiating of the function $\lambda$ with respect to $t$ and $x, \lambda_{t}$ and $\lambda_{x}$ can easily be obtained as,

and

$$
\lambda_{t}=-\frac{1}{2(1-\rho)} \lambda^{3} v,
$$

$$
\lambda_{x}=-\frac{1}{2(1-2 \rho)} \lambda^{3} w .
$$


Therefore,

$$
\begin{aligned}
p_{t}-\lambda p_{x} & =(v+\lambda w)_{t}-\lambda(v+\lambda w)_{x} \\
& =v_{t}-\lambda v_{x}+\lambda\left(w_{t}-\lambda w_{x}\right)+w\left(\lambda_{t}-\lambda \lambda_{x}\right) \\
& =v_{t}-\lambda^{2} w_{x}-\lambda\left(v_{x}-w_{t}\right)+w\left(\lambda_{t}-\lambda \lambda_{x}\right) \\
& =-\frac{1}{2(1-2 \rho)} \lambda^{3} w(v+\lambda w)=\frac{1}{4(1-2 \rho)} \lambda^{2}(q-p) p .
\end{aligned}
$$

We can prove (22) in the same way as above, and it is obvious that (21) is hold.

For the next lemma, consider

$$
r=p_{x}+\frac{1}{8(1-2 \rho)} \lambda p q \quad \text { and } \quad s=q_{x}-\frac{1}{8(1-2 \rho)} \lambda p q .
$$

Lemma 2.2. $r$ and s satisfy

$$
\begin{aligned}
& r_{t}-\lambda r_{x}=\frac{\lambda^{2}}{4(1-2 \rho)}(2 q-3 p) r+\frac{\lambda^{3}}{32(1-2 \rho)}(2 p-3 q) p q+\frac{\lambda^{3} p(q-p)}{32(1-2 \rho)^{2}}(p+5 q), \\
& s_{t}+\lambda s_{x}=\frac{\lambda^{2}}{4(1-2 \rho)}(2 q-3 p) r+\frac{\lambda^{3}}{32(1-2 \rho)}(2 p-3 q) p q+\frac{\lambda^{3} p(q-p)}{32(1-2 \rho)^{2}}(p+5 q) .
\end{aligned}
$$

Proof. Suppose

Hence, by a direct computation we can get

$$
L_{1}=\frac{\partial}{\partial t}-\lambda \frac{\partial}{\partial x} \quad \text { and } \quad L_{2}=\frac{\partial}{\partial t}+\lambda \frac{\partial}{\partial x} .
$$

$$
\begin{aligned}
& L_{1} p_{x}=\frac{1}{4(1-2 \rho)} \lambda^{2}\left((2 q-3 p) p_{x}+p q_{x}\right)+\frac{1}{8(1-2 \rho)^{2}} \lambda^{3} p(q-p)^{2}, \\
& L_{2} q_{x}=\frac{1}{4(1-2 \rho)} \lambda^{2}\left((2 p-3 q) q_{x}+q p_{x}\right)-\frac{1}{8(1-2 \rho)^{2}} \lambda^{3} q(p-q)^{2} .
\end{aligned}
$$

Now we can easily prove (23) and (23).

Notice from Cauchy problem (7), (16) and (19), we can write following equations at $t=0$

$$
\begin{aligned}
& p=p_{0}(x) \equiv u_{1}(x)+\lambda_{0}(x) u_{0}^{\prime}(x), \quad u=u_{0}(x) \\
& q=q_{0}(x) \equiv u_{1}(x)-\lambda_{0}(x) u_{0}^{\prime}(x),
\end{aligned}
$$

where $\lambda_{0}(x)=\sqrt{\frac{1-2 \rho}{u_{0}(x)}}$. Now in following theorem we show that the Cauchy problem (7) has a unique global solution under some conditions.

Theorem 2.3. Let $M_{1}$ be a positive constant satisfying

$$
0 \leq p(x, t) \leq M_{1} \quad \text { and } \quad 0 \leq q(x, t) \leq M_{1},
$$

then, on $D(T)$,

$$
|u(x, t)| \leq M(T), \quad\left|u_{x}(x, t)\right| \leq M(T), \quad\left|u_{t}(x, t)\right| \leq M(T),
$$


where $M(T)$ is a positive constant, and

$$
|r(x, t)| \leq M(T), \quad|s(x, t)| \leq M(T),
$$

$$
D(T)=\{(x, t) \mid x \in \mathbb{R}, 0 \leq t \leq T, T>0\} .
$$

Hence, the Cauchy problem (7) has a unique global classical solution on $t \geq 0$, by the local existence theorem of the classical solution to quasilinear hyperbolic systems.

Proof. Consider any point $(t, x)$, and let

$$
x=x_{1}\left(t, \beta_{1}\right), \quad x=x_{2}\left(t, \beta_{2}\right), \quad x=x_{3}\left(t, \beta_{3}\right)
$$

be the $\lambda_{1}, \lambda_{2}$ and $\lambda_{3}$ characteristics, respectively, that satisfy

$$
\begin{gathered}
\left(x_{1}\right)_{t}=\lambda_{1}=-\lambda, \quad\left(x_{2}\right)_{t}=\lambda_{2}=0, \quad\left(x_{3}\right)_{t}=\lambda_{3}=\lambda \\
x_{1}\left(0, \beta_{1}\right)=\beta_{1}, \quad x_{2}\left(0, \beta_{2}\right)=\beta_{2}, \quad x_{3}\left(0, \beta_{3}\right)=\beta_{3} .
\end{gathered}
$$

By integrating (21) along the $\lambda_{2}$ characteristics we can obtain

$$
u(x, t)=u_{0}\left(\beta_{2}\right)+\frac{1}{2} \int_{0}^{t}(p+q)\left(x_{2}\left(\tau, \beta_{2}\right), \tau\right) d \tau .
$$

Thus, as a result of (21), (27) and (28) we have

$$
\left|u_{t}\right| \leq M_{1}(T) \quad \text { and } \quad 0<\text { inf }_{x} u_{0}(x) \leq u(x, t) \leq M_{2}(T) .
$$

Using a same method, by integrating (23) along the $\lambda_{1}$ characteristics $x=x_{1}\left(t, \beta_{1}\right)$

$$
|r(x, t)| \leq M_{2}(T)+M_{3}(T) \int_{0}^{t} R(\tau) d \tau, \quad \text { where } R(t)=\sup _{x}|r(x, t)| .
$$

Therefore, we have $|r(x, t)| \leq M_{4}(T)$ by the Bellman lemma. As a similar way, holds $|s(x, t)| \leq M_{5}(T)$. Since $\left(u_{x}\right)_{t}=\frac{1}{2}(r+s)$, it is obvious that

$$
\left|u_{x}(x, t)\right| \leq M_{6}(T),
$$

which $M_{i}(t)$ for $i=1,2,3, \ldots$ denote positive constants.

\section{Proof of Theorem 1.2}

On the basis of the local existence and uniqueness theorems of the classical solutions to the quasilinear hyperbolic systems ([7]), to prove Theorem (1.2) it suffices to establish uniform a priori estimates of the $C^{1}$ norms of $p, q$ and $u$. We have following lemma from $[2,3]$.

Lemma 3.1. Suppose

$$
\begin{aligned}
u_{t}+\lambda_{1}(x, t) u_{x} & =A(x, t)(u-v), \\
v_{t}+\lambda_{2}(x, t) v_{x} & =B(x, t)(v-u),
\end{aligned}
$$

where $\lambda_{1}, \lambda_{2}, A$ and $B$ are continuous functions, and $\lambda_{1} \leq \lambda_{2}$. If $A$ and $B$ are both non positive, then

$$
\min \left(u_{0}(x), v_{0}(x)\right) \leq u(x, t), v(x, t) \leq \max \left(u_{0}(x), v_{0}(x)\right) .
$$

For prove Theorem 1.2 we need the following lemma. 
Lemma 3.2. On the existence domain of the classical solution to the Cauchy problem (7) and (25), if (10) and (11) hold, then

$$
\begin{aligned}
& 0 \leq p(x, t) \leq \sup _{x \in \mathbb{R}} p_{0}(x), \\
& 0 \leq q(x, t) \leq \sup _{x \in \mathbb{R}} q_{0}(x), \\
& 0<\inf _{x \in \mathbb{R}} u_{0}(x) \leq u(x, t) \leq \sup _{x} u_{0}(x)+C t,
\end{aligned}
$$

where $C>0$ is a constant.

Proof. Along $\lambda_{1}$ characteristics, we can obtain

$$
p(x, t)=p_{0}\left(\beta_{1}\right) \exp \left(\int_{0}^{t} \frac{1}{4(1-2 \rho)} \lambda^{2}(q-p)\left(x_{1}\left(\tau, \beta_{1}\right), \tau\right) d \tau\right) .
$$

By $(25)$ and $(10), p_{0}(x) \geq 0$ for all $x \in \mathbb{R}$. Therefore, we have $p(x, t) \geq 0$. In a similar way we can prove $q(x, t) \geq 0$. As a result of these inequalities, we have

$$
\frac{1}{4(1-2 \rho)} \lambda^{2} p \geq 0 \quad \text { and } \quad \frac{1}{4(1-2 \rho)} \lambda^{2} q \geq 0 .
$$

Hence, by Lemma 3.1 we can easily see that

$$
p(x, t) \leq \sup _{x} p_{0}(x) \quad \text { and } \quad q(x, t) \leq \sup _{x} q_{0}(x) .
$$

Also, we can get following equality by integrating (21)

$$
u(x, t)=u_{0}\left(\beta_{2}\right)+\frac{1}{2} \int_{0}^{t}(p+q)\left(x_{2}\left(\tau, \beta_{2}\right), \tau\right) d \tau .
$$

Thus, we can get to result since $p(x, t) \geq 0$ and $q(x, t) \geq 0$.

Proof of Theorem 1.2. Now from aforementioned Lemma 3.2 and Theorem 2.3, Theorem 1.2 is obvious.

Note. By (6) and (29), and based on the hypotheses of Theorem 1.2, we have

$$
|R(x, t)| \leq M_{7}(T)
$$

\section{Proof of Theorem 1.3}

The blow-up phenomena of the hyperbolic geometric flow will be discussed in this section.

Suppose

$$
m=\sqrt{\lambda} p \quad \text { and } \quad n=\sqrt{\lambda} q
$$

We have

$$
\frac{1}{4} \lambda^{2} q=\frac{1-2 \rho}{4}\left((\ln u)_{t}-\lambda(\ln u)_{x}\right) \quad \text { and } \quad \frac{1}{4} \lambda^{2} p=\frac{1-2 \rho}{4}\left((\ln u)_{t}+\lambda(\ln u)_{x}\right) \text {. }
$$

By the use of (16), (19) and Lemma 2.1 the following lemma can be proven 
Lemma 4.1. $m$ and $n$ satisfy

$$
\begin{aligned}
m_{t}-\lambda m_{x} & =-\frac{1}{4(1-2 \rho)} \lambda^{3 / 2} m^{2} \\
n_{t}-\lambda n_{x} & =-\frac{1}{4(1-2 \rho)} \lambda^{3 / 2} n^{2} .
\end{aligned}
$$

Observe that at $t=0$, set

$$
\begin{aligned}
& m=m_{0}(x)=\sqrt[4]{\frac{1-2 \rho}{u_{0}(x)}}\left(u_{1}(x)+\frac{u_{0}^{\prime}(x)}{\sqrt{u_{0}(x)}}\right), \\
& n=n_{0}(x)=\sqrt[4]{\frac{1-2 \rho}{u_{0}(x)}}\left(u_{1}(x)-\frac{u_{0}^{\prime}(x)}{\sqrt{u_{0}(x)}}\right) .
\end{aligned}
$$

Proof of Theorem 1.3. Without loss of generality, we assume that (12) holds; in the same way we can proceed if (13) holds.

As a result of (33) and (34) we have $m_{t}-\lambda m_{x} \leq 0$ and $n_{t}-\lambda n_{x} \leq 0$. Thus, we can easily see that

$$
m(x, t)+n(x, t) \leq M_{0} \quad \text { and } \quad M_{0} \equiv \operatorname{supm}_{0}(x)+\operatorname{supn}_{0}(x) .
$$

Notice that $u_{0}(x) \geq k>0$, and also from (12) and (35) we have $m_{0}(x)<0$. Next, the get following equation is obtained from (33) by integrating along $\lambda_{1}$ characteristics. That is,

$$
m\left(x_{0}, t\right)=m_{0}\left(x_{0}\right) / F\left(t, x_{0}\right)
$$

where

$$
F\left(t, x_{0}\right)=1+m_{0}\left(x_{0}\right) / 4(1-2 \rho) \int_{0}^{t} \lambda^{3 / 2}\left(x_{1}\left(x_{0}, \tau\right), \tau\right) d \tau \text { and } \lambda^{3 / 2}=\left(\frac{u}{1-2 \rho}\right)^{-3 / 4} \text {. }
$$

By $(21)$ and $(32)$, it is easy to see that $\left(\left(\frac{u}{1-2 \rho}\right)^{-3 / 4}\right)_{t}=\frac{3}{8(1-2 \rho)}(m+n)$. Hence, we have

$$
\left(\frac{u}{1-2 \rho}\right)^{3 / 4}(x, t)=\left(\frac{u_{0}}{1-2 \rho}\right)^{3 / 4}\left(x_{0}\right)+\frac{3}{8(1-2 \rho)} \int_{0}^{t}(m+n)\left(x_{2}\left(x_{0}, \tau\right), \tau\right) d \tau .
$$

By (4), (37) and (40), we get

$$
u^{3 / 4}(x, 0) \geq k^{3 / 4} \quad \text { and } \quad u^{3 / 4}(x, t) \geq M^{3 / 4}+\frac{3}{8(1-2 \rho)} M_{0} t .
$$

We consider three cases.

Case (i). If $M_{0}<0$, then there exists $\tau_{0}=8(1-2 \rho) M^{3 / 4} /\left(3\left(-M_{0}\right)\right)>0$, such that

$$
u(x, t) \leq 0 \quad \text { and } \quad t \geq \tau_{0} .
$$

This imply the system in (7) is meaningless for $t \geq \tau_{0}$, that is, it admits a unique local classical solution. 
Case(ii). If $M_{0}=0$, then, by (39) and (41), following inequality can be easily obtained

$$
F\left(x_{0}, t\right) \leq 1+\frac{1-2 \rho}{4} m_{0}\left(x_{0}\right) M^{-3 / 4} t .
$$

When $F\left(x_{0}, 0\right)=1>0$ and $m_{0}\left(x_{0}\right)<0$, we can find $t_{0}=4 M^{3 / 4} /(1-2 \rho)\left(-m_{0}\left(x_{0}\right)\right)>$ 0 , such that

$$
F\left(x_{0}, t\right) \rightarrow 0^{+} \quad \text { as } t \rightarrow t_{0}^{-} .
$$

Thus, the finite time $T=T\left(x_{0}\right)>0$ exists such that

$$
F\left(x_{0}, t\right) \rightarrow-\infty \quad \text { as } t \rightarrow T^{-} .
$$

Case(iii). If $M_{0}>0$, then, by (39) and (41) we can get

$$
F\left(x_{0}, t\right) \leq 1+\frac{2 m_{0}\left(x_{0}\right)}{3 M_{0}} \ln \left(1+\frac{3 M_{0}}{8(1-2 \rho) M^{3 / 4}} t\right) .
$$

Therefore, since, $F\left(x_{0}, 0\right)=1>0$ and $m_{0}\left(x_{0}\right)<0$, there exists $t_{*}>0$ such that $F\left(x_{0}, t\right) \rightarrow 0^{+}$as $t \rightarrow t_{*}^{-}$, and then (43) follows.

\section{Proof of Theorem 1.4} $R(x, t)$

In this section, we will study the asymptotic behaviour of the scalar curvature

Proof of Theorem 1.4. We assume that (15) holds. Using Lemmas 3.1 and 3.2, we have

$$
K_{1} \leq p(x, t) \leq K_{2} \quad \text { and } \quad K_{1} \leq q(x, t) \leq K_{2},
$$

where here after $C_{i}$ for $i=1,2, \ldots$ denote positive constants independent of $t$ and $x$. Therefore, we can obtain the following inequality as a result of (4) and (28)

$$
K_{3}(1+t) \leq u(x, t) \leq K_{4}(1+t),
$$

and then

$$
\frac{K_{3}^{3 / 4}(1+t)^{3 / 4}}{1-2 \rho} \leq \frac{u^{3 / 4}(x, t)}{1-2 \rho} \leq \frac{K_{4}^{3 / 4}(1+t)^{3 / 4}}{1-2 \rho} \leq \frac{K_{4}^{3 / 4}(1+t)}{1-2 \rho} .
$$

It follows from(40) and (44) that

$$
K_{5}(1+\ln (1+t)) \leq F(x, t) \leq K_{6}\left(1+(1+t)^{1 / 4}\right) .
$$

Thus, by (15) and (38), we get

$$
0 \leq \frac{K_{7}}{1+(1+t)^{1 / 4}} \leq m(x, t) \leq \frac{K_{8}}{1+\ln (1+t)} .
$$

Similarly, we can obtain

$$
0 \leq \frac{K_{7}}{1+(1+t)^{1 / 4}} \leq m(x, t) \leq \frac{K_{8}}{1+\ln (1+t)} .
$$

Hence, $m(x, t) \rightarrow 0$ and $n(x, t) \rightarrow 0$ as $t \rightarrow+\infty$. Noting (44), (45) and (46), we get $p=\frac{1}{\sqrt{\lambda}} m=\frac{u}{1-2 \rho}^{1 / 4} m, \quad q=\frac{1}{\sqrt{\lambda}} n=\frac{u}{1-2 \rho} n, \quad u_{x}=\frac{p-q}{2 \lambda}=\frac{1}{2} u^{3 / 4}(m-n)$, 
and

$$
-\frac{K_{8}}{1+\ln (1+t)} \leq m(x, t)-n(x, t) \leq \frac{K_{8}}{1+\ln (1+t)} .
$$

Then, we can easily obtain

$$
\left|u_{x}\right| \leq K_{9} \frac{(1+t)^{3 / 4}}{1+\ln (1+t)}
$$

Next an easy derivation gives

$$
u_{x x}=\frac{p_{x}-q_{x}}{2 \lambda}+\frac{1}{2} \lambda^{2} u_{x}^{2}=\frac{1}{2} u^{1 / 2}\left(p_{x}-q_{x}\right)+\frac{1}{2 u} u_{x}^{2} .
$$

Let $\bar{p}=p u$ and $\bar{q}=q u$. Thus, based on [5] we have

$$
\begin{aligned}
& L_{1} \overline{p_{x}}=-A_{1} \overline{p_{x}}-B_{1} \overline{q_{x}}, \\
& L_{2} \overline{q_{x}}=-A_{2} \overline{p_{x}}-B_{2} \overline{q_{x}},
\end{aligned}
$$

where

$$
A_{1}=\frac{1}{4}(2 \bar{q}+3 \bar{q}), \quad B_{1}=\frac{3}{4} \bar{q}, \quad A_{2}=\frac{3}{4} \bar{q}, \quad B_{2}=\frac{1}{4}(2 \bar{p}+3 \bar{q}) .
$$

Therefore, by [5] holds

$$
\left|\bar{p}_{x}(x, t)\right|,\left|\bar{q}_{x}(x, t)\right| \leq K_{10} .
$$

Noting that $p_{x}-q_{x}=u_{x}(p-q) / u+u\left(\overline{p_{x}}-\overline{q_{x}}\right)$, and from (6) and (49), we have

$$
R=\frac{1}{u^{3}}\left(u_{x}^{2}-u u_{x x}\right)=\frac{u_{x}^{2}}{2 u^{3}}-\frac{p_{x}-q_{x}}{2 u^{3 / 2}}=\frac{u_{x}^{2}}{2 u^{3}}-\frac{\overline{p_{x}}-\overline{q_{x}}}{2 u^{1 / 2}}-\frac{u_{x}(m-n)}{2 u^{3}} .
$$

Thus, from (41), (47), (48), (50) and (51) we conclude that

$$
|R(x, t)| \leq \frac{K_{11}}{(1+\ln (1+t))^{2}(1+t)^{3 / 2}}+\frac{K_{1} 2}{(1+t)^{1 / 2}}+\frac{K_{13}(1+t)^{3 / 4}}{(1+t)^{3}(1+\ln (1+t))^{2}} .
$$

Hence, $R(x, t) \rightarrow 0$ as $t \rightarrow+\infty$.

Acknowledgement. The authors thank the referee for valuable sugestions.

\section{REFERENCES}

[1] Catino, G., Cremaschi, Djadli, L.Z., Mantegazza C., and Mazzieri, L., "The RicciBourguignon flow", Pacific Journal of Mathematics, 287(2)(2017), 337-370.

[2] Hong, J.X., "The global smooth solutions of Cauchy problems for hyperbolic equation of Monge-Ampére type", Nonlinear Anal. 24(1995),1649-1663.

[3] Kong, D.X., "Maximum principle in nonlinear hyperbolic systems and its applications", Nonlinear Anal. 32(7)(1998), 871-880.

[4] Kong D.X. and Liu, K., "Wave character of metrics and hyperbolic geometric flow", J. Math. Phys. 48(10)(2007),103508.

[5] Kong, D.X., Liu K., and Xu, D.L., "The hyperbolic geometric flow on Reimann surfaces", Comm. Partial Differential Equations, 34(4-6)(2009), 553-580.

[6] Li T.T. and Liu, F.G., "Singularity caused by eigenvalues for quasilinear hyperbolic systems", Comm. Partial Diffrential Equations, 28(3-4)(2003),477-503. 
[7] Li T.T. and Yu, W.C., Boundary value problems for quasilinear hyperbolic systems, Duke University Math. Series 5, Duke University Press, Durham, NC, 1985.

[8] Liu F. and Zhang, Y., "Global classical solutions to hyperbolic geometric flow on Riemann surfaces", Pacific Journal of Mathematics, 246(2)(2010), 333-343. 